\title{
Surface and optical properties of ethylene glycol-based nanofluids containing silicon dioxide nanoparticles: an experimental study
}

\author{
Julian Traciak ${ }^{1} \cdot$ Jolanta Sobczak $^{1} \cdot$ Rafał Kuzioła $^{2} \cdot$ Joanna Wasąg $^{2} \cdot$ Gaweł Żyła $^{1}$ D
}

Received: 26 March 2021 / Accepted: 3 September 2021 / Published online: 9 October 2021

(c) The Author(s) 2021

\begin{abstract}
In this paper, the results of the experimental study on density, surface tension and optical properties of ethylene glycol-based nanofluids containing silicon dioxide have been presented. The nanofluid-air surface tension values were determined in the temperature range from $283.15 \mathrm{~K}$ to $318.15 \mathrm{~K}$ with Du Noüy ring method with the commercial equipment and self-made station. To determine the surface tension of a fluid, the information about its density is required; therefore, density of all examined nanofluids was measured in the same temperature range, and results were summarized in the paper. The refractive index of $\mathrm{SiO}_{2}$-EG nanofluids was determined in the same temperature range in which the surface tension was examined. Finally, the extinction (UV-Vis spectra) of these materials was examined and presented.
\end{abstract}

Keywords Nanofluid $\cdot$ Silicon dioxide $\cdot$ Ethylene glycol $\cdot$ Surface tension $\cdot$ Optical properties

\section{Introduction}

Nanofluids, which are suspensions of nanoparticles (with at least one diameter less than $100 \mathrm{~nm}$ ), exhibit unique physical properties as it was summarized in some recent review papers [1-6]. This kind of properties makes nanofluids an interesting material for the numerous possible applications [7-11]. One of the promising possible application of nanofluids, based on its complex optical properties, is using them in the renewable energy industry, especially in solar collectors and PV/T systems [12-18]. Beside the fact that nanofluids are known for over 25 years know, the scientific community still does not have a coherent theoretical model describing the physical properties of nanofluids. Numerous scientific teams tries to develop theoretical models; however, such works require detailed, reliable experimental data which in many cases are missing in the literature. This paper brings a contribution on this field and presents results of

Gaweł Żyła

gzyla@prz.edu.pl

1 Department of Experimental Physics, Rzeszow University of Technology, Rzeszow, Poland

2 Department of Materials Science, Faculty of Natural Sciences and Health, Institute of Engineering and Technical Sciences, The John Paul II Catholic University of Lublin, Lublin, Poland experimental study on density, surface tension and optical properties of ethylene glycol (EG) based nanofluids containing silicon dioxide $\left(\mathrm{SiO}_{2}\right)$ nanoparticles.

The physical properties of ethylene glycol-based nanofluids containing $\mathrm{SiO}_{2}$ were already the subject of research. Zyła and Fal [19] presented results of the study on the dynamic viscosity, thermal and electrical conductivity of $\mathrm{SiO}_{2}$-EG nanofluids containing up to 5.0 mass\% of nanoparticles at $298.15 \mathrm{~K}$. They showed that in examined mass fraction range nanofluids exhibit Newtonian nature and thermal conductivity could be described with classical Maxwell model. Li et al. [20] examined the dependence of thermal conductivity of $\mathrm{SiO}_{2}$-EG nanofluids on the time of sonication during preparation process. The specific heat of various types of nanofluids with $\mathrm{SiO}_{2}$ nanoparticles was examined by Akilu et al. [21]. It was summarized that there is a decrease of nanofluids specific heat with increasing mass fraction of nanoparticles in a $\mathrm{SiO}_{2}$-EG nanofluids. Recently, Alazwari et al. [22] used ANN and RSM techniques to evaluate the thermal conductivity of $\mathrm{SiO}_{2}-\mathrm{EG}$ nanofluids and possible applications of it. Fal et al. deepened the understanding of $\mathrm{SiO}_{2}$-EG properties with information on the effect of temperature on electrical conductivity [23]. Additionally, they developed a ethylene glycol-based silicon oxide-lignin nanofluids, and presented a comprehensive study on its electrical and dielectric properties [24]. 
According to the best of the authors' knowledge, there are no literature data on the surface tension of $\mathrm{SiO}_{2}$-EG nanofluids and there are only a few papers on the surface tension (ST) of ethylene glycol-based nanofluids available in the literature. Moosavi et al. [25] show an increase in surface tension of ZnO-EG nanofluids with increasing volume concentration of nanoparticles. Harikrishnan et al. [26] used pendant drop method to measure surface tension of $\mathrm{Bi}_{2} \mathrm{O}_{3}$ -EG and CuO-EG at $303.15 \mathrm{~K}$. They observed that the surface tension of the tested nanofluids did not increase with the mass concentration of nanoparticles in suspension. Wanic et. al. [27] presented results of surface tension for the pendant drop and Du Noüy method for TiN-EG, AlN-EG and $\mathrm{Si}_{3} \mathrm{~N}_{4}$ -EG nanofluids and both methods show that ST increases slightly with the concentration of nanoadditions.

The optical properties of nanofluids are interesting from the point of view of possible applications of such materials and from the point of view of basic science. Again, according to the best authors' knowledge $\mathrm{SiO}_{2}$-EG nanofluids were not subject of the evaluation of optical properties. However, one might find some papers on the optical properties of other ethylene glycol-based nanofluids. Żyła et al. [28] showed that for $\mathrm{Si}_{3} \mathrm{~N}_{4}$-EG nanofluids, the transmittance of the nanofluid increases with increasing wavelength and decreases sharply with increasing nanoparticles volume fraction. Sani et. al. [29] studied nanodiamond particles in ethylene glycol and showed a much lower transmittance than pure base fluid, leading to high sunlight absorption by these nanofluids with promising potential in direct absorption solar collectors. Di Rosa et al. [30] examined optical properties of EG-based nanofluids containing nanodiamonds with two various purities. Torres et. al. [31] compared samples of TiN-EG nanofluid prepared with pulse laser ablation in liquids (PLAL) and with two-step method. It was presented that the nanofluid synthesized by PLAL showed a lower transmission spectrum for the visible wavelengths. The refractive index of ethylene glycol-based nanofluids was subject of studies performed by Żyła et al. [28] and Sobczak et al. [32] for $\mathrm{Si}_{3}$ $\mathrm{N}_{4}$-EG and carbon black nanofluids, respectively.

In this experimental work, the surface and optical properties of $\mathrm{SiO}_{2}$-EG nanofluids with mass fraction change from 1 mass\% to 5 mass\% at the temperature range of $283.15-318.15 \mathrm{~K}$ were investigated to fill this gap in nanofluids research.

\section{Materials and methods}

In this section, the sample preparation and measuring procedures were described along with relative uncertainty values.

\section{Sample preparation}

The nanoparticles used in this study were manufactured by PlasmaChem GmbH (Berlin, Germany); according to manufacturer declaration, nanopowder exhibit $99.8 \%$ purity. The detailed information, about physical properties of this material, was presented elsewhere [19]. Figure 1 shows the scanning electron microscope (SEM) picture of used nanoparticles.

In the experiments ethylene glycol (EG), with purity over 99\% produced by Fisher Chemical (Loughborough, UK), was employed as a base fluid. All samples were prepared in $50 \mathrm{~mL}$ vials using two-step method. Firstly, the specific amount, which was weighted on an analytical balance Pioneer Semi-Micro PX225DM (Ohaus, Poland) with an accuracy of $0.01 \mathrm{mg}$, of nanopowder was putted into the vial. The second step of the preparation consisted adding the correct amount of EG in order to obtain the right concentration. After that, sample was mechanical mixed on IKA Vortex 3 shaker (IKA, Staufen, Germany) for 30 minutes. In order to ensure well dispersion of nanoparticles and avoid agglomerates for a period of 200 min samples were kept in Emmi $60 \mathrm{HC}$ ultrasonic water bath (EMAG, Moerfelden-Walldorf, Germany). The final step included immersion each sample in a high-energy ultrasonic probe (Sonics, Vibracell VCX 130, Sonics \& Materials Inc., Newtown, USA) for 5 minutes. The samples were prepared in various mass concentrations of the particles from 1 to 5 mass\% with 1 mass\% step. During preparation process, no surfactants were used and the

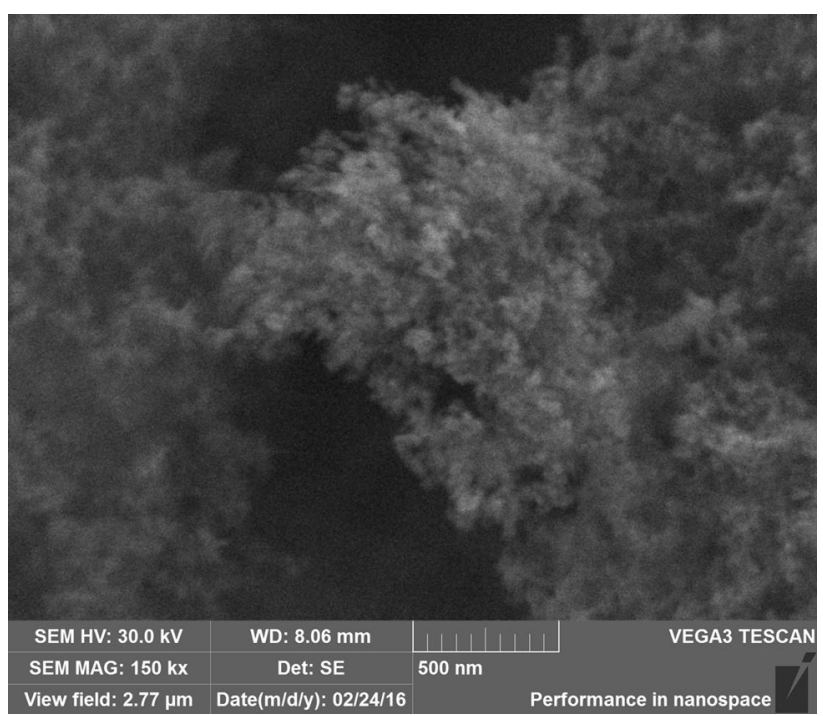

Fig. 1 Scanning electron microscope (SEM) picture of dry $\mathrm{SiO}_{2}$ nanoparticles. Reproduced with permission from Ref. [19], license number 5080150950041 
measurements were conducted immediately after sample preparation.

\section{Fourier Transform Infrared (FTIR) measurements}

Samples were analyzed by using a Thermo Scientific Nicolet iS5 with ATR iD5, with resolution of $4 \mathrm{~cm}^{-1}$, in a range of wave number from 4000 to $400 \mathrm{~cm}^{-1}$ and with a scan number of 64. The attenuated total reflection (ATR) is a technique of infrared spectroscopy. The ATR analysis is based on internal reflection at a diamond with high refractive index placed in direct contact with the sample. The radiation passes through the diamond and is refracted. Then, it strikes the sample several times and penetrates for about $2 \mu \mathrm{m}$ and is reflected, recrossing the crystal and reach the detector DLaTGS.

\section{Mass density measurements}

Density measurements of the studied nanofluids were performed using an automatic oscillating U-tube density meter DMA 4100 M (Anton Paar, Graz, Austria). The nanofluid samples were measured over a temperature range of $285.15-320.15 \mathrm{~K}$ with a step of $5 \mathrm{~K}$. Three measurement series were conducted for each temperature with ten measurements in each one. The uncertainty of the measured values derived from this instrument was determined by the deviation between the experimental data and the corresponding value in the literature and the standard deviation of one hundred measurement points of distilled water at $298.15 \mathrm{~K}$. For these one hundred measurements, the water mass density obtained was $0.9969 \mathrm{~g} \mathrm{~cm}^{-3}$ with a standard deviation of $0.0002 \mathrm{~g} \mathrm{~cm}^{-3}$. The literature [33] states that the density of water is $0.997066 \pm 0.000001 \mathrm{~g} \mathrm{~cm}^{-3}$. Taking into account the literature values and the achieved experimental results, the relative uncertainty of the density measurements can be determined as $0.1 \%$.

\section{Surface tension measurements}

Surface tension measurements were performed on a PIMT1A tensiometer (Polon-Izot, Warsaw, Poland). To measure surface tension, this device uses the Du Noüy ring method. The surface tension of the studied nanofluids was conducted in the temperature range $285.15-320.15 \mathrm{~K}$ with a step of $5 \mathrm{~K}$. Ten surface tension measurements were taken for each temperature and the given experimental values were calculated as the average. The uncertainty of this device was determined as $0.03 \mathrm{mNm}^{-1}$ [27]. Taking into account all these factors, the relative uncertainty was estimated as $1 \%$. Additionally the results obtained with the commercial device at the room temperature were compared with home-made tensiometer construction of which is presented elsewhere [34].

\section{Refractive index measurements}

The refractive index was determined using Refractometer Abbemat 550 (Anton Paar OptoTec GmbH, Seelze-Letter, Germany). After setting the air reference in measuring chamber a $500 \mu \mathrm{L}$ of a sample was placed with HTL Discovery Comfort DV1000 single channel pipette (HTL Lab Solution, Warsaw, Poland) — an accuracy of volume is $0.5 \%$. The value of the refractive index was calculated based on the average of three series of measurements. The relative uncertainty of used device is less than $1 \%$ as it was presented elsewhere [28].

\section{UV-Vis measurements}

Light extinction of silicon dioxide-ethylene glycol nanofluids was measured with a UV-Vis spectrometer Evolution 220 (ThermoFisher SCIENTIFIC, Waltham, MA, USA) with an absorbance range of $190 \mathrm{~nm}$ to $1100 \mathrm{~nm}$. The research was carried out with inspection range from 190 to $800 \mathrm{~nm}$ with a $1 \mathrm{~nm}$ gap and an integration time of $0.30 \mathrm{~s}$. The scanning speed was set to $200.00 \mathrm{~nm} \mathrm{~min}^{-1}$. The tested samples were contained in $3 \mathrm{~mL}$ quartz cuvettes.

\section{Results}

In this section, the results of measurements of fundamental physical properties of $\mathrm{SiO}_{2}$-EG nanofluids at the temperature of $298.15 \mathrm{~K}$ were presented and discussed. The complete results of measurements in the temperature range 283.15-308.15K are included Appendix 1.

\section{FTIR}

Figure 2 shows the FTIR spectra of $\mathrm{SiO}_{2}$-EG and pure ethylene glycol as a reference sample. All samples show the identical characteristic spectra for ethylene glycol, therefore analyzed spectra show vibrations: $3299 \mathrm{~cm}^{-1}$ (OH group), $2937 \mathrm{~cm}^{-1}\left(\mathrm{CH}_{2} / \mathrm{CH}_{3}\right.$ group $), 1410 \mathrm{~cm}^{-1}\left(\mathrm{CH}_{3}\right.$ group $)$, $1366 \mathrm{~cm}^{-1}\left(\mathrm{CH}_{3} / \mathrm{CH}_{2}\right.$ group $), 1083 \mathrm{~cm}^{-1}\left(\mathrm{CH}_{2} / \mathrm{CH}\right.$ group$)$. This proves that the samples are stable and no new chemical bonds are formed.

\section{Mass density}

The Table 1 shows the obtained mass density values of nanofluids with different concentrations at $298.15 \mathrm{~K}$. The base fluid density measurements stand in high agreement 


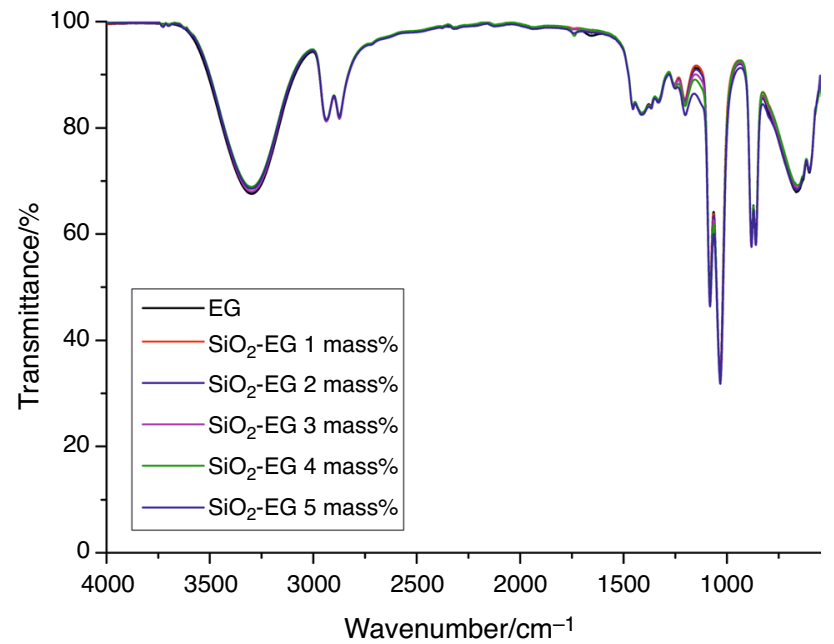

Fig. 2 Fourier transform infrared (FTIR) spectra of $\mathrm{SiO}_{2}$-EG nanofluids and pure ethylene glycol

with our previous works [32, 35]. In Fig. 3, a linear increase in the density of the nanosuspension can be observed with increasing mass fraction of nanoparticles.

More than 20 years ago, Pak and Cho [36] proposed the following equation to describe this dependence:

$\rho_{\mathrm{nf}}=\left(1-\varphi_{\mathrm{V}}\right) \rho_{\mathrm{bf}}+\varphi_{\mathrm{V}} \rho_{\mathrm{p}}$,

where $\rho_{\mathrm{nf}}$ is density of nanofluid, $\rho_{\mathrm{bf}}$ is density of the base fluid and $\rho_{\mathrm{p}}$ is density of particles, $\varphi_{\mathrm{V}}$ is the volume fraction corresponding to the mass fraction $\varphi_{\mathrm{m}}$ by:

$\varphi_{\mathrm{V}}=\frac{\varphi_{\mathrm{m}}}{\rho_{\mathrm{p}}\left(\frac{\varphi_{\mathrm{m}}}{\rho_{\mathrm{p}}}+\frac{1-\varphi_{\mathrm{m}}}{\rho_{\mathrm{bf}}}\right)}$.

This correlation was based on ideal gas mixing theory. According to the authors, this equation allows to model the

Table 1 Experimental values of the density, $\rho$, surface tension, $\gamma$, and refractive index, $n$, of $\mathrm{SiO}_{2}$-EG nanofluids at pressure $p=0.10 \mathrm{MPa}$ and temperature $T=298.15 \mathrm{~K}$ for various mass fractions $\varphi_{\mathrm{m}}$, and volume fractions $\varphi_{\mathrm{V}}$ of nanoparticles

\begin{tabular}{lllll}
\hline$\varphi_{\mathrm{m}} /-$ & $\varphi_{\mathrm{V}} /-$ & $\rho_{\mathrm{nf}} / \mathrm{g} \mathrm{cm}^{-3}$ & $\gamma_{\mathrm{nf}} / \mathrm{mN} \mathrm{m}^{-1}$ & $n_{\mathrm{nf}} /-$ \\
\hline 0.00 & 0.000 & 1.1098 & 47.926 & 1.43025 \\
0.01 & 0.005 & 1.1152 & 48.280 & 1.43047 \\
0.02 & 0.010 & 1.1202 & 48.142 & 1.43061 \\
0.03 & 0.015 & 1.1265 & 48.290 & 1.43077 \\
0.04 & 0.020 & 1.1319 & 48.325 & 1.43092 \\
0.05 & 0.026 & 1.1375 & 48.328 & 1.43106 \\
\hline
\end{tabular}

The estimated standard relative uncertainty $u_{\mathrm{r}}\left(\varphi_{\mathrm{m}}\right)=0.01$, $u_{\mathrm{r}}\left(\varphi_{\mathrm{V}}\right)=0.001, u_{\mathrm{r}}(\rho)=0.001, u_{\mathrm{r}}(\gamma)=0.01, u_{\mathrm{r}}(n)=0.01$, $u(p)=0.01 \mathrm{MPa}$ and $u(T)=0.10 \mathrm{~K}$
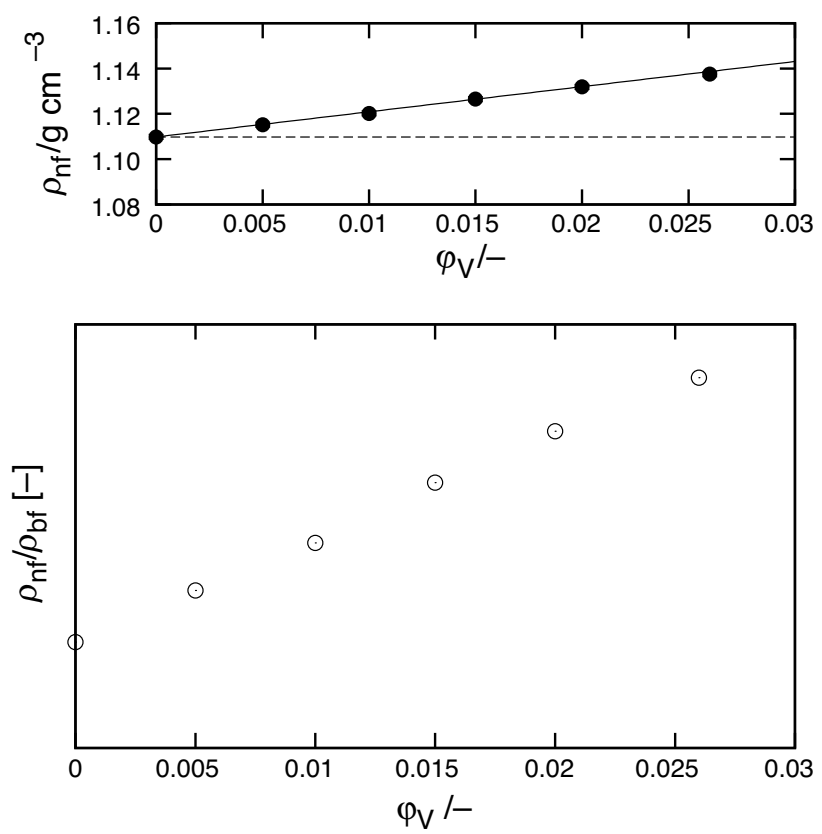

Fig. 3 Dependence of density, $\rho$, and density ratio, $\rho_{\mathrm{nf}} / \rho_{\mathrm{bf}}$, on the volume fraction, $\varphi_{\mathrm{V}}$, of the particles for the $\mathrm{SiO}_{2}$-EG nanofluids at 298.15 K. Dotted line presents the value of pure ethylene glycol, solid line shows function (1)
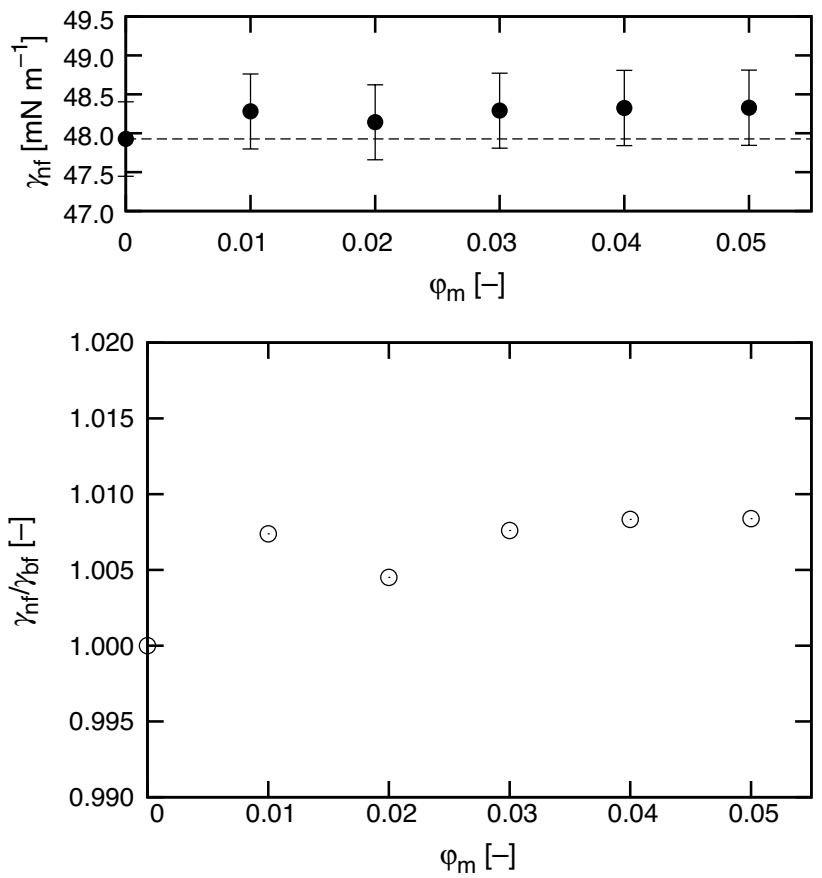

Fig. 4 Dependence of surface tension, $\gamma$, and surface tension ratio, $\gamma_{\mathrm{nf}}$ $/ \gamma_{\mathrm{bf}}$, on the mass fraction, $\varphi_{\mathrm{m}}$, of the particles for the $\mathrm{SiO}_{2}$-EG nanofluids at $298.15 \mathrm{~K}$. Dotted line presents the value of surface tension of pure ethylene glycol 
density of nanofluids within a few percent accuracy. The experimental results obtained for $\mathrm{SiO}_{2}$-EG are consistent with this model as can be seen in Fig. 3.

\section{Surface tension}

The surface tension values of $\mathrm{SiO}_{2}$-EG nanofluids at $298.15 \mathrm{~K}$ are presented in Table 1 . The addition of nanoparticles to the base fluid slightly affects the surface tension, which can be observed in Fig. 4. However, the data obtained are within the measurement uncertainty for the literature value of ethylene glycol surface tension $48.02 \mathrm{mNm}^{-1}$ [37]. Despite an increase in the mass concentration of the nanoparticles, the surface tension emerges around $48.3 \mathrm{mNm}^{-1}$. Based on these data, it can be concluded that the addition of $\mathrm{SiO}_{2}$ nanoparticles at the concentrations tested to ethylene glycol does not affect the surface tension of the base fluid significantly.

Additionally, the surface tension of the samples was examined with a tensiometer built using arduino and 3D printing technology. The construction and its operation is presented in the recent paper [34]. Surface tension measurements were performed at $298.15 \pm 2.00 \mathrm{~K}$, and the obtained results of the measurements performed with this device are shown in Fig. 5. Taking into account, the

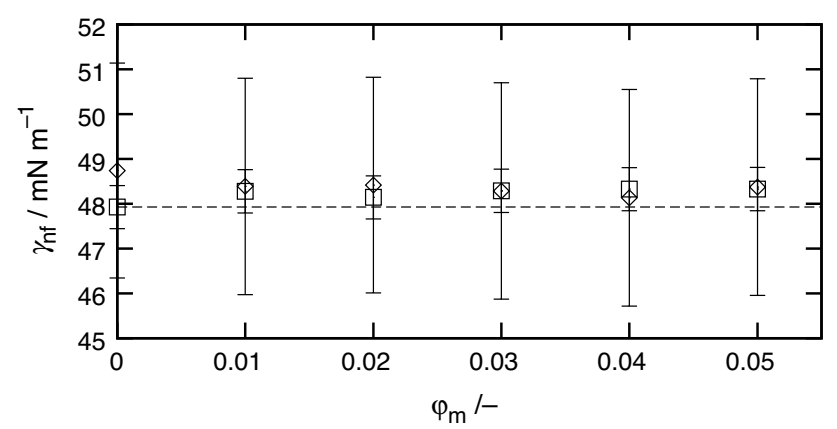

Fig. 5 Dependence of surface tension, $\gamma$, on the mass fraction, $\varphi_{\mathrm{m}}$, of the particles for the $\mathrm{SiO}_{2}$-EG nanofluids at $298.15 \mathrm{~K}$. Square points present results obtained with commercial equipment, diamondsresults obtained with self-made station. Dotted line presents the value of surface tension of pure ethylene glycol measured with commercial equipment measurement uncertainties, one could note that the results of measurements of the surface tension observed with this tensiometer stands in high agreement with measurements made on a commercial device.

Absence or slight increase in surface tension is also observed for other nanoparticles in ethylene glycol based nanofluids such as nitrides $[27,38]$ or oxides $[25,26]$.

\section{Refractive index}

Despite the great interest in the thermophysical properties of nanofluids containing $\mathrm{SiO}_{2}$, there is lack of information about their optical properties designated in the experimental way. However, in some publications, this topic is presented from the theoretical perspective $[39,40]$. The obtained results of refractive index, $n$, analyzed for $\mathrm{SiO}_{2}$ -EG nanofluids with different concentrations of nanoparticles are summarized in Table 2. The measured value of refractive index increases with the higher mass fraction of
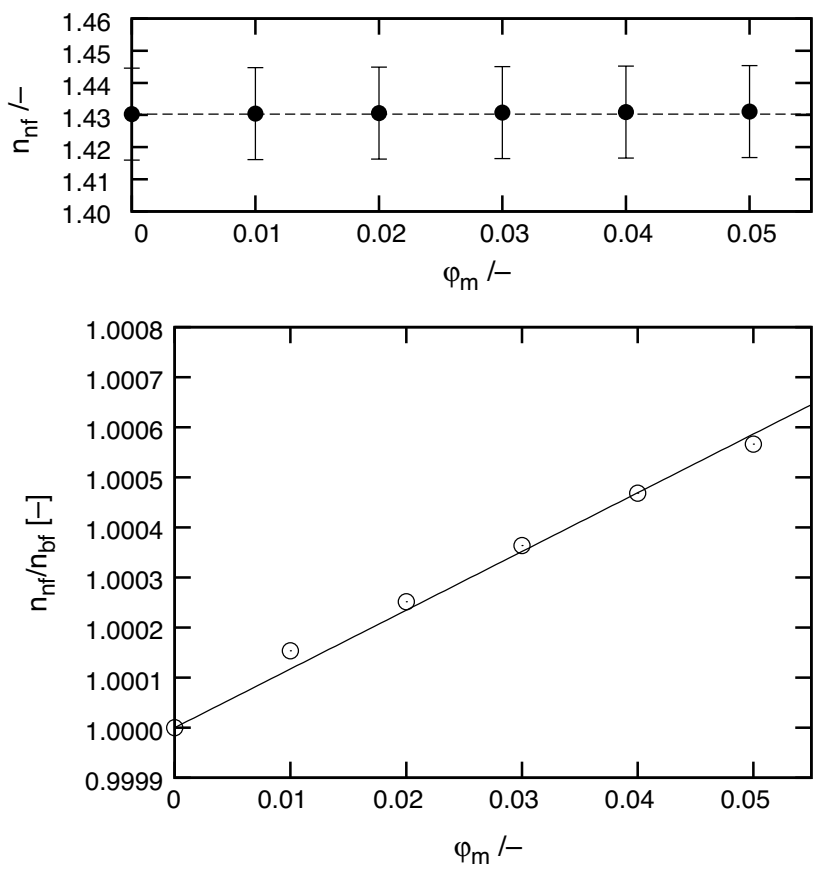

Fig. 6 Dependence of refractive index, $n$, and refractive index ratio, $n_{\mathrm{nf}} / n_{\mathrm{bf}}$, on the mass fraction, $\varphi_{\mathrm{m}}$, of the particles for the $\mathrm{SiO}_{2}-\mathrm{EG}$ nanofluids at $298.15 \mathrm{~K}$. Dotted line presents the value of refractive index of pure ethylene glycol, solid line shows Eq. (3) 
$\mathrm{SiO}_{2}$ nanoparticles in the sample. Using the least squares fitting method, in which correlation coefficient, $R^{2}$, is 1 , a linear function was fitted to the data according to the equation:

$\frac{n_{\mathrm{nf}}}{n_{\mathrm{bf}}}=1+0.01173 \varphi_{\mathrm{m}}$

Furthermore, the graph in Fig. 6 depicts fitted linear function to the experimentally determined values of $n$.

\section{Extinction}

Figure 7 shows the extinction spectra obtained for silicon dioxide-ethylene glycol nanofluids with various mass concentration of nanoparticles. A maximum extinction peak was observed at a wavelength of about $200 \mathrm{~nm}$. As it could be noted, with an increasing amount of $\mathrm{SiO}_{2}$ nanoparticles, the light extinction of $\mathrm{SiO}_{2}$-EG nanofluid increased until it reached a maximum value for concentration 5 mass\%. The maximum extinction for an increasing mass concentration of the nanofluid increase from 0.79 to 1.70 , respectively. The fewer particles dispersed in the solution, the lower the light extinction of the nanofluids. The presence of nanoparticles increases the scattering and therefore the extinction. This proves that even a greater amount of $\mathrm{SiO}_{2}$ does not cause the particles to easily aggregate in suspension, reducing the extinction of particle light during UV-Vis measurements. Additionally, the stability of these nanofluids is confirmed by the fact that the extinction peak

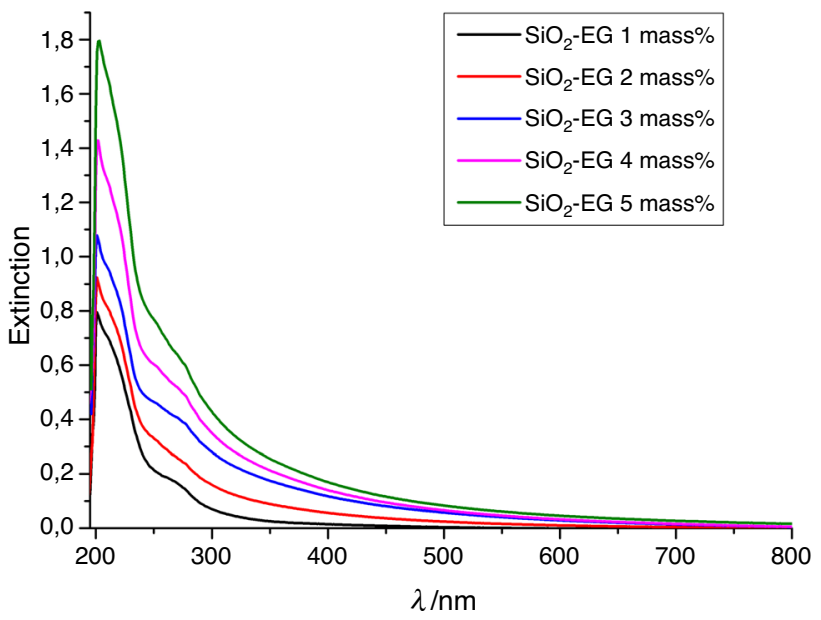

Fig. 7 Extinction spectra for $\mathrm{SiO}_{2}$-EG nanofluids with various mass concentration of nanoparticles

varies to a small extent in the entire range of the tested concentrations. In colloidal solutions, not the absorbance value is measured, but the absorption and dispersion due to the presence of nanoparticles. Therefore, in this case, the concept of extinction is more appropriate. The presence of nanoparticles increases the scattering and therefore the extinction.

\section{See Appendix Fig. 8 and Table 2}


Fig. 8 Dependence of a density, b surface tension, $\mathbf{c}$ refractive index on the mass fraction, $\varphi_{\mathrm{m}}$, of the particles for the $\mathrm{SiO}_{2}$-EG nanofluids at temperature range of $283.15-318.15 \mathrm{~K}$
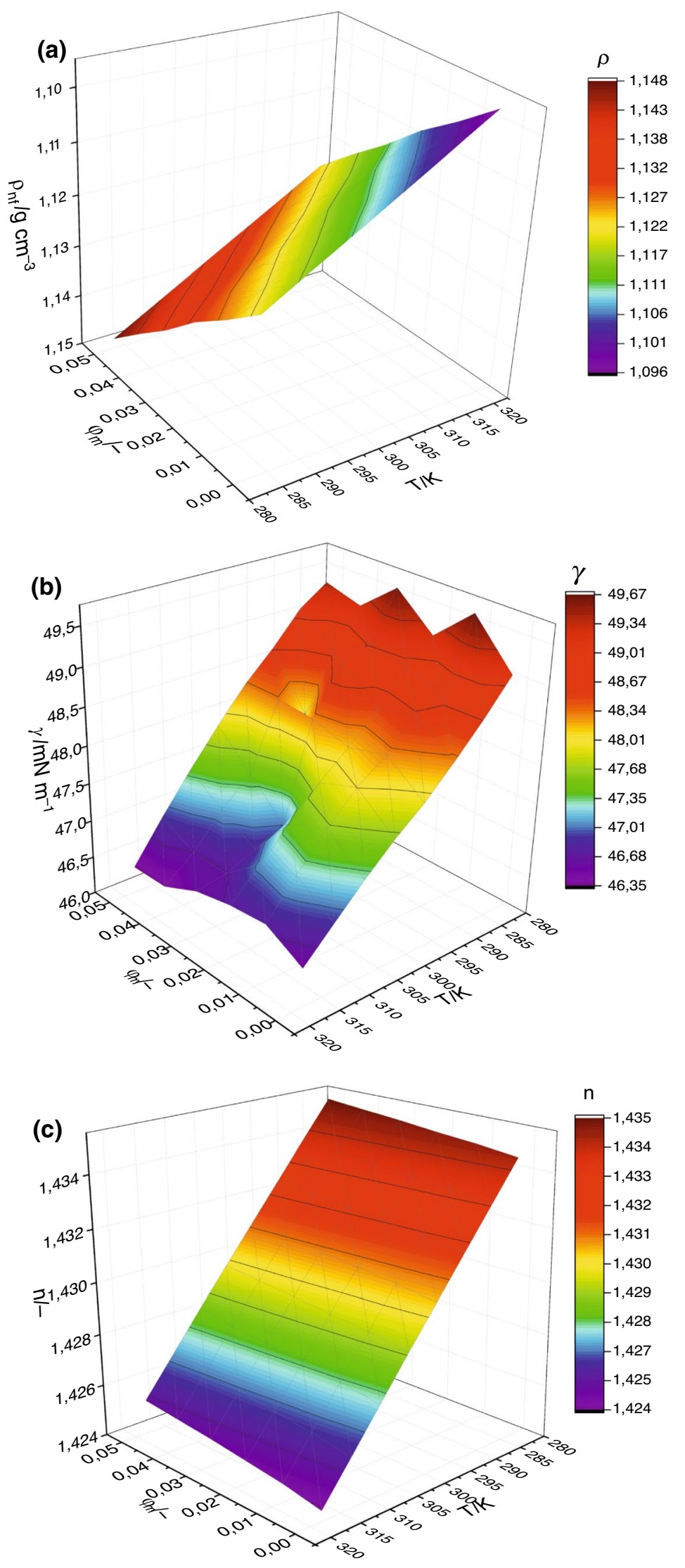
Table 2 Experimental values of the density, $\rho$, surface tension, $\gamma$, and refractive index, $n$, of $\mathrm{SiO}_{2}$-EG nanofluids at pressure $p=0.10 \mathrm{MPa}$ and various temperatures from $T=283.15 \mathrm{~K}$ to $T=318.15 \mathrm{~K}$ and different fractions of nanoparticles: mass, $\varphi_{\mathrm{m}}$, and volume, $\varphi_{\mathrm{V}}$

\begin{tabular}{llllllllll}
\hline & $T / \mathrm{K} \rightarrow$ & 283.15 & 288.15 & 293.15 & 298.15 & 303.15 & 308.15 & 313.15 & 318.15 \\
\hline$\varphi_{\mathrm{m}} /-$ & $\varphi_{\mathrm{V}} /-$ & $\rho / \mathrm{g} \mathrm{cm}^{-3}$ & & & & & & & \\
0.00 & 0.000 & 1.1202 & 1.1167 & 1.1133 & 1.1098 & 1.1063 & 1.1028 & 1.0992 & 1.0957 \\
0.01 & 0.005 & 1.1257 & 1.1222 & 1.1187 & 1.1152 & 1.1118 & 1.1082 & 1.1047 & 1.1012 \\
0.02 & 0.010 & 1.1307 & 1.1272 & 1.1237 & 1.1202 & 1.1167 & 1.1132 & 1.1097 & 1.1062 \\
0.03 & 0.015 & 1.1369 & 1.1334 & 1.1300 & 1.1265 & 1.1229 & 1.1194 & 1.1159 & 1.1123 \\
0.04 & 0.020 & 1.1423 & 1.1388 & 1.1354 & 1.1319 & 1.1284 & 1.1249 & 1.1213 & 1.1177 \\
0.05 & 0.026 & 1.1480 & 1.1445 & 1.1410 & 1.1375 & 1.1340 & 1.1305 & 1.1270 & 1.1235 \\
$\varphi_{\mathrm{m}} /-$ & $\varphi_{\mathrm{V}} /-$ & $\gamma / \mathrm{mN} \mathrm{m})^{-1}$ & & & & & & & \\
0.00 & 0.000 & 49.109 & 48.678 & 48.286 & 47.926 & 47.624 & 47.273 & 46.890 & 46.504 \\
0.01 & 0.005 & 49.670 & 48.707 & 48.657 & 48.280 & 48.014 & 47.646 & 47.218 & 46.758 \\
0.02 & 0.010 & 49.210 & 48.946 & 48.490 & 48.142 & 47.823 & 47.442 & 47.069 & 46.704 \\
0.03 & 0.015 & 49.632 & 49.024 & 48.895 & 48.290 & 47.015 & 46.837 & 46.669 & 46.595 \\
0.04 & 0.020 & 49.229 & 48.704 & 48.003 & 48.325 & 47.459 & 47.116 & 46.679 & 46.370 \\
0.05 & 0.026 & 49.365 & 49.109 & 48.694 & 48.328 & 47.883 & 47.406 & 46.901 & 46.357 \\
$\varphi_{\mathrm{m}} /-$ & $\varphi_{\mathrm{V}} /-$ & $n /-$ & & & & & & & \\
0.00 & 0.000 & 1.43455 & 1.43312 & 1.43169 & 1.43025 & 1.42882 & 1.42734 & 1.42586 & 1.42440 \\
0.01 & 0.005 & 1.43472 & 1.07498 & 1.43189 & 1.43047 & 1.42904 & 1.42759 & 1.42613 & 1.42468 \\
0.02 & 0.010 & 1.43484 & 1.43343 & 1.43202 & 1.43061 & 1.42919 & 1.42775 & 1.42630 & 1.42486 \\
0.03 & 0.015 & 1.43499 & 1.43358 & 1.43218 & 1.43077 & 1.42936 & 1.42792 & 1.42648 & 1.42504 \\
0.04 & 0.020 & 1.43511 & 1.43372 & 1.43232 & 1.43092 & 1.42950 & 1.42806 & 1.42662 & 1.42518 \\
0.05 & 0.026 & 1.43524 & 1.43386 & 1.43246 & 1.43106 & 1.42965 & 1.42823 & 1.42677 & 1.42532 \\
\hline
\end{tabular}

The estimated standard relative uncertainty $u_{\mathrm{r}}\left(\varphi_{\mathrm{m}}\right)=0.01, u_{\mathrm{r}}\left(\varphi_{\mathrm{V}}\right)=0.001, u_{\mathrm{r}}(\rho)=0.001, u_{\mathrm{r}}(\gamma)=0.01$, $u_{\mathrm{r}}(n)=0.01, u(p)=0.01 \mathrm{MPa}$ and $u(T)=0.10 \mathrm{~K}$

\section{Conclusions}

The paper summarizes results of the experimental study on the surface and optical properties of $\mathrm{SiO}_{2}$-EG nanofluids. The mass density, surface tension, refractive index and extinction of the nanofluids with various mass fractions have been examined. It was presented that mass density could be modeled with well-known Pak and Cho correlation. In case of surface tension, it was presented that fraction of the nanoparticles have marginal influence on this property; the surface tension of nanofluids increases after adding the nanoparticles, but taking into account the uncertainty of measurements this increase is insignificant. The refractive index increase with the nanoparticle fraction and could be described with linear function. It was also presented that in these nanofluids absorbance of light is the highest for wavelength approximately $200 \mathrm{~nm}$, and its value depends on the mass fraction of the particles. This paper brings a contribution to the knowledge of the physical properties of the $\mathrm{SiO}_{2}$-EG; however, one could note that there are still some gaps in this area. Future research could be focused on the heat capacity, contact angle and heat transfer of this type of nanofluids.

Acknowledgements Research was partially supported by Rzeszow University of Technology grant no. PB27.FE.21.001.
Open Access This article is licensed under a Creative Commons Attribution 4.0 International License, which permits use, sharing, adaptation, distribution and reproduction in any medium or format, as long as you give appropriate credit to the original author(s) and the source, provide a link to the Creative Commons licence, and indicate if changes were made. The images or other third party material in this article are included in the article's Creative Commons licence, unless indicated otherwise in a credit line to the material. If material is not included in the article's Creative Commons licence and your intended use is not permitted by statutory regulation or exceeds the permitted use, you will need to obtain permission directly from the copyright holder. To view a copy of this licence, visit http://creativecommons.org/licenses/by/4.0/.

\section{References}

1. Murshed SS, Estellé P. A state of the art review on viscosity of nanofluids. Renew Sustain Energy Rev. 2017;76:1134-52.

2. Mahian O, Kolsi L, Amani M, Estellé P, Ahmadi G, Kleinstreuer C, Marshall JS, Siavashi M, Taylor RA, Niazmand H, et al. Recent advances in modeling and simulation of nanofluid flows-part I: Fundamentals and theory. Phys Rep. 2019;790:1-48.

3. Qiu L, Zhu N, Feng Y, Michaelides EE, Żyła G, Jing D, Zhang X, Norris PM, Markides CN, Mahian O. A review of recent advances in thermophysical properties at the nanoscale: from solid state to colloids. Phys Rep. 2020;843:1-81.

4. Estellé P, Cabaleiro D, Żyła G, Lugo L, Murshed SS. Current trends in surface tension and wetting behavior of nanofluids. Renew Sustain Energy Rev. 2018;94:931-44.

5. Minea AA. A review on electrical conductivity of nanoparticleenhanced fluids. Nanomaterials. 2019;9(11):1592. 
6. Fal J, Mahian O, Żyła G. Nanofluids in the service of high voltage transformers: breakdown properties of transformer oils with nanoparticles, a review. Energies. 2018;11(11):2942.

7. Sajid MU, Ali HM. Recent advances in application of nanofluids in heat transfer devices: a critical review. Renew Sustain Energy Rev. 2019;103:556-92.

8. Olabi A, Elsaid K, Sayed ET, Mahmoud MS, Wilberforce T, Hassiba RJ, Abdelkareem MA. Application of nanofluids for enhanced waste heat recovery: A review. Nano Energy. 2021;84:105871.

9. Sidik NAC, Samion S, Ghaderian J, Yazid MNAWM. Recent progress on the application of nanofluids in minimum quantity lubrication machining: A review. Int $\mathbf{J}$ Heat Mass Transf. 2017;108:79-89.

10. Sidik NAC, Yazid MNAWM, Mamat R. A review on the application of nanofluids in vehicle engine cooling system. Int Commun Heat Mass Transf. 2015;68:85-90.

11. Murshed SS, de Castro CN. Conduction and convection heat transfer characteristics of ethylene glycol based nanofluids-a review. Appl energy. 2016;184:681-95.

12. Alawi OA, Kamar HM, Mallah A, Mohammed HA, Kazi S, Sidik NAC, Najafi G. Nanofluids for flat plate solar collectors: Fundamentals and applications. J Clean Prod. 2020;291:125725.

13. Olfian H, Ajarostaghi SSM, Ebrahimnataj M. Development on evacuated tube solar collectors: A review of the last decade results of using nanofluids. Solar Energy. 2020;211:265-82.

14. Sheikholeslami M, Farshad SA, Ebrahimpour Z, Said Z. Recent progress on flat plate solar collectors and photovoltaic systems in the presence of nanofluid: A review. J Clean Prod. 2021;293:126119.

15. Wahab A, Hassan A, Qasim MA, Ali HM, Babar H, Sajid MU. Solar energy systems-potential of nanofluids. J Mol Liq. 2019;289:111049.

16. Tiwar AK, Kumar V, Said Z, Paliwal H. A review on the application of hybrid nanofluids for parabolic trough collector: Recent progress and outlook. J Clean Prod. 2021;292:126031.

17. Said Z, Hachicha AA, Aberoumand S, Yousef BA, Sayed ET, Bellos E. Recent advances on nanofluids for low to medium temperature solar collectors: energy, exergy, economic analysis and environmental impact. Prog Energy Combust Sci. 2021;84:100898.

18. Trong Tam N, Viet Phuong N, Hong Khoi P, Ngoc Minh P, Afrand M, Van Trinh P, Hung Thang B, Żyła G, Estellé P. Carbon nanomaterial-based nanofluids for direct thermal solar absorption. Nanomaterials. 2020;10(6):1199.

19. Żyła G, Fal J. Viscosity, thermal and electrical conductivity of silicon dioxide-ethylene glycol transparent nanofluids: An experimental studies. Thermochimica Acta. 2017;650:106-13.

20. Li Y, Kalbasi R, Nguyen Q, Afrand M. Effects of sonication duration and nanoparticles concentration on thermal conductivity of silica-ethylene glycol nanofluid under different temperatures: an experimental study. Powder Technol. 2020;367:464-73.

21. Akilu S, Baheta A, Sharma K, Said M. Experimental determination of nanofluid specific heat with $\mathrm{SiO}_{2}$ nanoparticles in different base fluids. In: AIP Conference Proceedings. 2017. vol. 1877(1), p. 090001.

22. Alazwari MA, Abu-Hamdeh NH, Salilih EM. Exergetic performance analysis on helically coiled tube heat exchanger-forecasting thermal conductivity of $\mathrm{SiO}_{2} / \mathrm{EG}$ nanofluid using ann and rsm to examine effectiveness of using nanofluids. J Therm Anal Calorim. 2021;144:1-13.

23. Fal J, Żyła G. Effect of temperature and mass concentration of $\mathrm{SiO}_{2}$ nanoparticles on electrical conductivity of ethylene glycol. Acta Phys Pol A. 2017;132(1):155-7.
24. Fal J, Wanic M, Budzik G, Oleksy M, Żyła G. Electrical conductivity and dielectric properties of ethylene glycol-based nanofluids containing silicon oxide-lignin hybrid particles. Nanomaterials. 2019;9(7):1008.

25. Moosavi M, Goharshadi EK, Youssefi A. Fabrication, characterization, and measurement of some physicochemical properties of $\mathrm{ZnO}$ nanofluids. Int J Heat Fluid Flow. 2010;31(4):599-605.

26. Harikrishnan A, Dhar P, Agnihotri PK, Gedupudi S, Das SK. Effects of interplay of nanoparticles, surfactants and base fluid on the surface tension of nanocolloids. Eur Phys J E. 2017;40(5):1-14.

27. Wanic M, Cabaleiro D, Hamze S, Fal J, Estellé P, Żyła G. Surface tension of ethylene glycol-based nanofluids containing various types of nitrides. J Therm Anal Calorim. 2020;139(2):799-806.

28. Żyła G, Fal J, Bikić S, Wanic M. Ethylene glycol based silicon nitride nanofluids: An experimental study on their thermophysical, electrical and optical properties. Phys E Low-dimensional Syst Nanostruct. 2018;104:82-90.

29. Sani E, Papi N, Mercatelli L, Żyła G. Graphite/diamond ethylene glycol-nanofluids for solar energy applications. Renew Energy. 2018;126:692-8.

30. Di Rosa D, Wanic M, Fal J, Żyła G, Mercatelli L, Sani E. Optical and dielectric properties of ethylene glycol-based nanofluids containing nanodiamonds with various purities. Powder Technol. 2019;356:508-16.

31. Torres-Mendieta R, Mondragón R, Puerto-Belda V, MendozaYero O, Lancis J, Juliá JE, Mínguez-Vega G. Characterization of tin/ethylene glycol solar nanofluids synthesized by femtosecond laser radiation. ChemPhysChem. 2017;18(9):1055-60.

32. Sobczak J, Vallejo JP, Traciak J, Hamze S, Fal J, Estellé P, Lugo L, Żyła G. Thermophysical profile of ethylene glycol based nanofluids containing two types of carbon black nanoparticles with different specific surface areas. J Mol Liq. 2021;326:115255.

33. Steckel F, Szapiro S. Physical properties of heavy oxygen water. part 1. density and thermal expansion. Trans Faraday Soc. 1963;59:331-43.

34. Traciak J, Fal J, Żyła G. 3D printed measuring device for the determination the surface tension of nanofluids. Appl Surface Sci. 2021;561:149878149878.

35. Żyła G, Vallejo JP, Fal J, Lugo L. Nanodiamonds-ethylene glycol nanofluids: experimental investigation of fundamental physical properties. Int J Heat Mass Transf. 2018;121:1201-13.

36. Pak BC, Cho YI. Hydrodynamic and heat transfer study of dispersed fluids with submicron metallic oxide particles. Exp Heat Transf Int J. 1998;11(2):151-70.

37. Haynes WM. CRC handbook of chemistry and physics. Boca Raton: CRC Press; 2014.

38. Żyła G, Fal J, Estellé P. Thermophysical and dielectric profiles of ethylene glycol based titanium nitride (TiN-EG) nanofluids with various size of particles. Int J Heat Mass Transf. 2017;113:1189-99.

39. Huang Z, Bai J, Luo P. Investigation on optical theoretical models of $\mathrm{SiO}_{2}$ nanofluids. In: International symposium on mechanical engineering and material science (ISMEMS 2017). Atlantis Press, 2017. pp. 108-113

40. Chai JY, Wong BT. Study of light scattering by $\mathrm{TiO}_{2}, \mathrm{Ag}$, and $\mathrm{SiO}_{2}$ nanofluids with particle diameters of 20-60 nm. J Nano Res. 2019;60:1-20.

Publisher's Note Springer Nature remains neutral with regard to jurisdictional claims in published maps and institutional affiliations. 